\title{
OMAE2014-24067
}

\section{TIDAL CURRENT TURBINE WAKE AND PARK LAYOUT IN TRANSIENT ENVIRONMENTS}

\author{
Matthias Arnold, Po Wen Cheng \\ Stuttgart Wind Energy (SWE) \\ University Stuttgart, Germany
}

\author{
Philipp Daus, Frank Biskup \\ Voith Hydro Ocean Current Technologies \\ Heidenheim, Germany
}

\begin{abstract}
Due to orbital velocities of the waves, the characteristics of tidal current turbines change over time. Therefore the induction factor and subsequent the wake is time dependent. Within the present research this time dependency is investigated by using Computational Fluid Dynamics (CFD) with Virtual Free Surface (VFS) and Actuator Disc (ACD) models.

Based on this setup several different wave and current scenarios are simulated and analyzed with respect to the transient velocities in the turbine wake. Special respect is taken to the velocity undulations radiated by the changing apparent velocities in the rotor plane. These undulations move with the wake of the tidal current turbines and increase wave loads on $2^{\text {nd }}$ row turbines in a park.

This paper presents an efficient method for simulation of wave and park interactions and investigates dynamic turbine wakes under a large variety of parameters. Based on these simulations a suggestion for a tidal park design with respect to a balance of fatigue loads and power output is concluded.
\end{abstract}

KEYWORDS: Tidal Current Turbine, Actuator Disc (ACD), Dynamic Wake, Wave induced Loads, Dynamic Inflow, Velocity Undulations, Computational Fluid Dynamics (CFD), Park Layout

\section{INTRODUCTION}

Loads on tidal current turbines are influenced by a wide range of environmental impacts. Surface waves, turbulence, marine growth, structural and turbine wakes to name some of them. These have been investigated by a wide range of researchers.

However the knowledge of interaction of these impacts is limited. Within the present research, the changes in turbine wake based on the environmental impact of surface waves are analyzed.

\subsection{VELOCITY UNDULATIONS}

During a wave period the velocity apparent to a tidal current turbine oscillates between a minimal and maximal value. Therefore the load and power output of the turbine oscillates coherently. This is coupled to a variation of the pressure drop $\Delta p$ resp. the velocity induction $\Delta v=v_{1}-v_{3}$.

Based on these variations in the induced velocity areas with increased and decreased velocity far behind the turbine $v_{3}$ is to be expected. These areas are moving with the wake velocity of the turbine, while the waves move with the wave speed. Together these 2 moving velocity fields form undulations in the wake velocity by destructive and productive superposition of the fields. This is equivalent to a displacement of energy within the wake and an energy exchange between the wave and the wake, leading to increased wave loads to turbines placed in a park within the wake of a previous row.

\subsection{LITERATURE REVIEW}

The interaction of waves with tidal current turbines has been investigated in a large number of publications. These can be grouped into the categories of loads and wakes. While the loads are investigated in the most cases by means of numerical simulation, [5], the wake is often measured in wave flumes, [12], [14]. The numerical prediction of the actual wake decay is difficult, [13], [16], and needs to be adjusted by empirical approximations.

However these experimental investigations experience the problem of limited depth of analysis, e.g. the flow velocity can be recorded only for single locations using Acoustic Doppler Velocimeter (ADV), [12]. To analyze the velocity field the experiment needs to be repeated for each location of measurement. Therefore the synchronization of data and subsequent the deduction of a momentary velocity field is difficult.

Based on this limited output the results of wake investigation are limited to the decay of the wake, [11], the 
increase in turbulence intensity, [4], and the change of these under influence of waves, [14]. The actual formation of the wake and large scale transients of the wake with waves as described in 1.1 have not been investigated yet and accounted to be covered by the turbulence intensity $I=\sigma(u)$.

The above described effect of velocity undulation and wake formation was shown in [7] and [9], but both of these publications focus on another aspect of the tidal current turbine simulation and therefore miss a detailed investigation of the behavior and influencing parameters. Therefore the present paper addresses a more detailed numerical analysis of this effect of velocity undulations.

\section{METHODOLOGY}

The above described phenomena are modeled within this research by means of an Actuator Disc in a 2-dimensional CFD simulation. Within this section the details of this model are given and the model is checked for validity.

\subsection{COMPUTATIONAL FLUID DYNAMICS (CFD)}

Within this research, the flow characteristics are simulated with the state of the art CFD-code Ansys CFX v14.5. It solves the Unsteady Reynolds Averaged Navier Stokes equations (URANS) by using the Finite Volume Method. Due to its implicit iteration scheme it is independent from time steps, stable and can therefore be used for simulation of larger timescales. Here the time resolution is defined to be fine enough for an detailed post-processing with $d t=\frac{\tau_{\text {Wave }}}{60}$.

\subsubsection{GOVERNING EQUATIONS}

The URANS solved within the CFD are a set of partial differential equations based on the conservation of mass, momentum and energy. This set can be further simplified by assumptions of a temperature independent incompressible fluid with density $\rho$ as done here and shown in Eq. (1), (2), [1], with velocity vector $\vec{u}$, pressure $p$, stress tensor $\tau$ and momentum source $S$. While $\vec{u}$ and $p$ are part of the solution, $\tau$ needs to be calculated by a mathematical model. Within this research $\tau$ is calculated with the SST turbulence model defined by [10].

$$
\begin{aligned}
& \nabla \cdot \vec{u}=0 \\
& \frac{\partial \vec{u}}{\partial t}+\nabla \cdot(\vec{u} \times \vec{u})=(-\nabla p+\nabla \cdot \tau+S) \cdot \frac{1}{\rho}
\end{aligned}
$$

\subsubsection{BOUNDARY CONDITIONS}

The computational domain is enclosed by a set of boundary conditions as shown in Fig. 1. The complete mathematical description of these boundary conditions can be found in [1] and not copied here for brevity. The Virtual Free Surface (VFS) addition to the opening condition is described in detail in 2.5 .

The fluid within the domain was defined to be 'water' with density $\rho=997 \frac{\mathrm{kg}}{\mathrm{m}^{3}}$ and dynamic viscosity $\eta=890 \cdot 10^{-6} \frac{\mathrm{kg}}{\mathrm{m} \cdot \mathrm{s}}$.

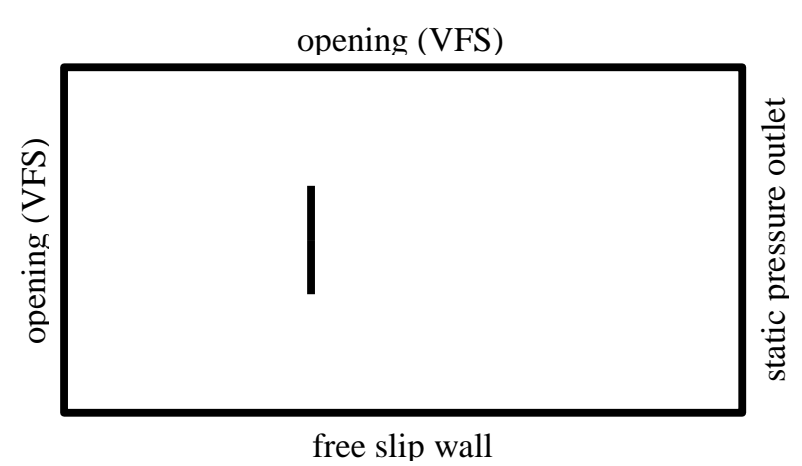

Fig. 1: Boundary conditions of computational domain

\subsection{ACTUATOR DISC THEORY (ACD)}

There are two basic approaches for the simulation of tidal rotors in CFD. On one hand, a full 3-dimensional geometric representation, on the other hand, a reduced model, the Actuator Disc (ACD), can be used. Due to the superior efficiency for wake simulations the ACD is used within this research. This ACD does not take the actual geometry of the rotor into account but applies a source term $S_{A C D}$ to the URANS equivalent to the rotor thrust loading defined by the pressure drop $\Delta p$. This source term is spread over a volume representing the cross section of the rotor with the finite thickness $\Delta x_{A C D}$ as shown in Eq. (3).

$$
S_{A C D}=\frac{d p}{d x}=\frac{\Delta p}{\Delta x_{A C D}}
$$

For the ACD multiple versions are available, ranging from a porous medium, [13], to the Blade Element Momentum Theory (BEMT), [2], [3]. Within the present research, the $c_{x^{-}}$ ACD and $F_{x}$-ACD are of relevance and detailed here. Both use an axial source term only and therefore can be used for 2 dimensional simulations.

Common to both is the underlying definition of the rotor behavior. A tidal turbine decelerates the fluid from the undisturbed flow $v_{1}$ to the velocity in the rotor disc $v_{2}$ and the full developed wake velocity $v_{3}$. The non-dimensional ratio of these is the induction factor a, Eq. 4, which can be used to calculate the thrust coefficient $c_{t}$, Eq. 5, of the rotor, Eq. 6 .

$$
\begin{aligned}
& a=\frac{v_{1}-v_{2}}{v_{1}}=\frac{v_{1}-v_{3}}{2 \cdot v_{1}} \\
& c_{t}=\frac{F_{x}}{\frac{\rho}{2} v_{1}^{2} \cdot \pi R_{\text {Rotor }}^{2}} \\
& c_{t}=4 \cdot a \cdot(1-a)
\end{aligned}
$$

\subsection{1. $c_{x}$-ACD}

The $c_{x}$-ACD calculates the thrust of the rotor based on a local thrust coefficient and the axial flow conditions within each numerical location. By assuming a constant axial velocity $u$ axial in the ACD, the source value for the URANS can be calculated. 


$$
\Delta p=\frac{\rho}{2} \cdot u^{2} \cdot c_{x}
$$

Based on the momentum theory of Betz the value $c_{x}$ can be derived from the thrust coefficient $c_{t}$, Eq. (8), resp. the induction factor $a$, Eq. (9), of the rotor.

$$
\begin{aligned}
& c_{x}=c_{t} \cdot\left(\frac{2}{1+\sqrt{1-c_{t}}}\right)^{2} \\
& c_{x}=\frac{4 \cdot a}{1-a}
\end{aligned}
$$

Based on this description of the source term, the $c_{x}$-ACD adapts its flow behavior to the surrounding fluid. It therefore behaves like a tidal current turbine with an infinite fast control, holding the optimal thrust coefficient constant relative to the apparent flow.

\subsection{2. $\quad F_{x}$-ACD}

The $F_{x}$-ACD assumes the rotor thrust to be constant over time and space. As shown in Eq. (10) the pressure drop is calculated based on the definition of the thrust coefficient $c_{t}$ and the free stream Eulerian velocity $v_{1}$.

$$
\Delta p=\frac{\rho}{2} \cdot v_{1}^{2} \cdot c_{t}
$$

\subsection{TURBULENCE}

Any gradient within a viscid velocity field causes turbulent disturbances. This small scale turbulence causes larger structures within the velocity field, as the wake of a turbine or the investigated velocity undulations, to decay over time. Therefore an adequate representation of the turbulence is required. The turbulence is simulated with the SST turbulence model, [10].

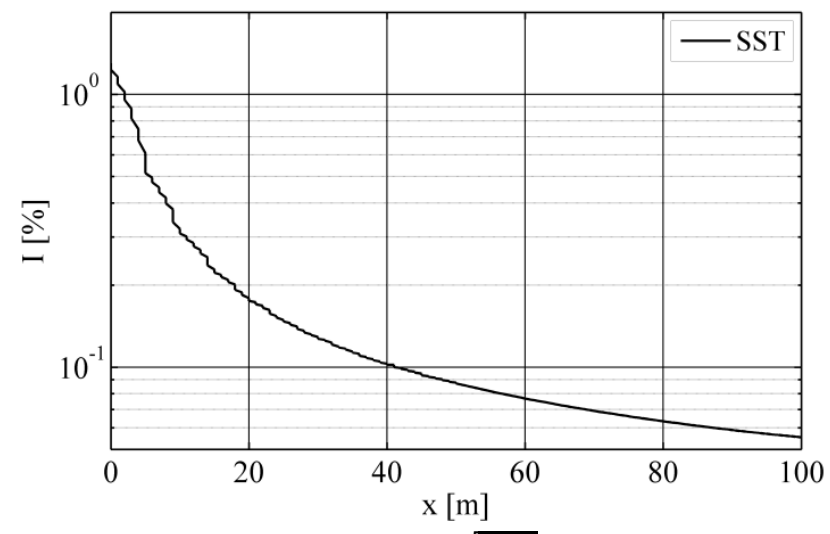

Fig. 2: Turbulence intensity $I=\bar{u} \cdot \sqrt{\frac{2}{3} \cdot k}$ in the inflow for a SST simulation

However in the present simulations the SST model leads to an unphysical small Turbulence Kinetic Energy (TKE) $k$ in the vicinity of the ACD due to the turbulence damping terms within the model as shown in Fig. 2. Therefore an additional source term $S_{\text {turb }}$ for the turbulence is required to maintain realistic inflow turbulence. This can be either limited to the volume of ACD as proposed by [3] or applied to the full fluid domain as a floor value for ambient turbulence, [15].

\subsubsection{ACD TURBULENCE SOURCE}

The turbulence intensity $I$ in the wake of a rotor has been investigated by [4]. Based on these findings, a change in turbulence intensity $\Delta I$ and TKE $\Delta k$ over the ACD can be calculated and the source term $S_{\text {turb }}$ is be defined by Eq. (13).

$$
\begin{aligned}
& \Delta I=c_{\text {turb }} \cdot a \\
& \Delta k=\frac{3}{2} \cdot(\Delta I \cdot u)^{2} \\
& S_{\text {turb }}=\Delta k \cdot \frac{\rho \cdot u}{\Delta x_{A C D}}
\end{aligned}
$$

The factor $c_{\text {turb }}$ within the empirical approximation was derived by [4] from measurements of a wind turbine to be $c_{t u r b}=0.725$. It can be adjusted to fit other turbine geometries or experimental data.

\subsubsection{AMBIENT TURBULENCE}

The floor value for ambient turbulence approach is based on the assumption, that in a real free stream the decay of TKE is in balance with turbulence sources due to e.g. ground roughness and thermal imbalances. These sources are not modeled within a CFD simulation. Therefore the balance converges to an unphysical small value of TKE. To overcome this problem, a minimum value for the turbulence intensity $I_{\text {floor }}$ is defined and preserved by a source value in each location of the simulation defined by Eq. (

$$
S_{\text {turb }}=\left\{\begin{array}{cc}
k_{p} \cdot\left(I_{\text {floor }}-I\right) & I<I_{\text {floor }} \\
0 & \text { else }
\end{array}\right.
$$

The factor $k_{p}$ is a proportional gain factor and can be adjusted to result in a fast but stable simulation. Within the present research a value of $k_{p}=5 \frac{\mathrm{kW}}{\mathrm{m}^{-3}}$ was suitable.

\subsubsection{VALIDATION OF THE WAKE}

For validation of the turbulence modeling the wake of an ACD in free stream has been simulated with both approaches. The dimensions of the 2-dimensional model are given in Fig. 3.

The results for this investigation are shown in Fig. 4 and compared to experimental results of [14] for the centerline velocity deficit $a_{d e f}$, Eq. (15). Both approaches do not match the peak near wake velocity deficit seen in the measurements. The comparison to an equivalent laminar simulation reveals that this is due to the formation of the wake deficit behind the ACD. A reason for this might result from the comparison of 3dimensional measurements within a flume to a 2-dimensional simulation with infinite extension. Also the effect of the nacelle within the experiment is unclear. 


$$
a_{d e f}=1-\frac{u}{v_{1}}
$$

The ambient turbulence approach predicts the damping of the velocity deficit within an acceptable range. Also the peak increase of the turbulence intensity on centerline matches within margin. This option is therefore used within the further research.

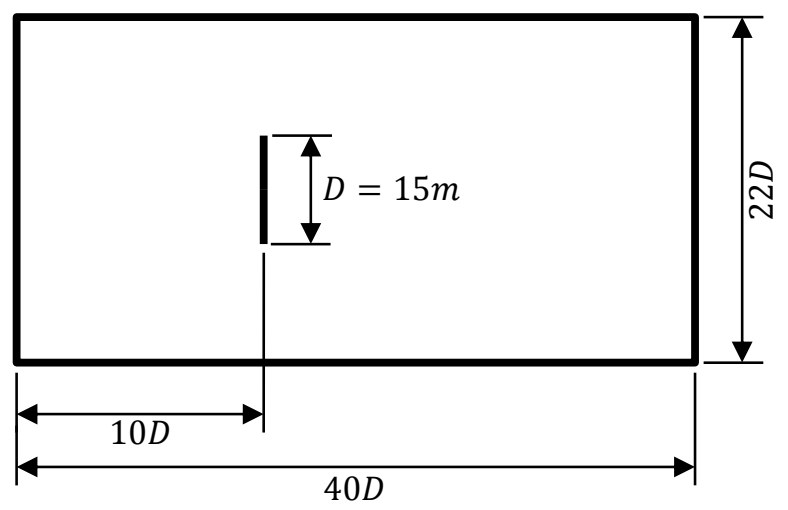

Fig. 3: Geometry of domain for 2D wake verification

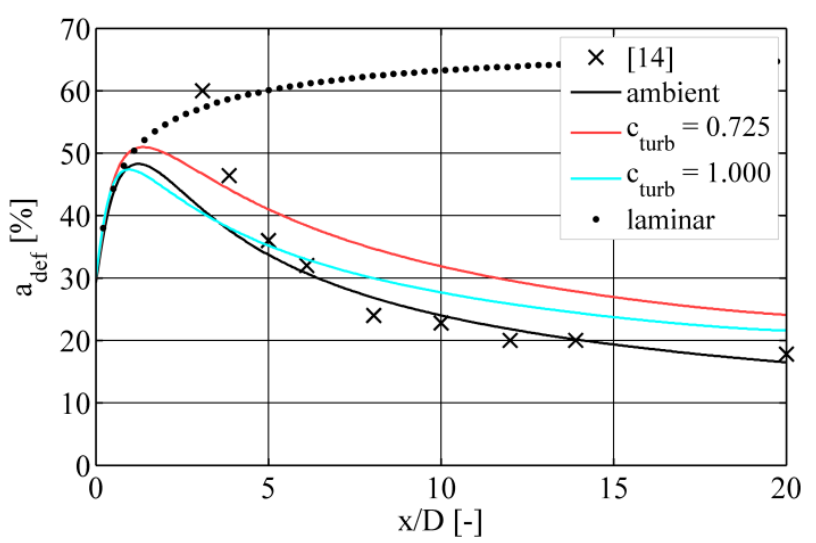

Fig. 4: Centerline wake deficit with $c_{x}=2$ and $I=10 \%$

\subsection{WAVE THEORY}

The wave theory describes the velocity $\vec{u}_{\text {wave }}$ and pressure field $p_{\text {wave }}$ of gravity waves on a free surface based on various assumptions. Within this research the linear Airy-wave and the Fenton-wave, [6], are used. Both are capable of simulating deep and shallow water waves, but differ in their field of application.

While the Airy-wave is a linear solution of the potential flow, the Fenton-wave is a stream function theory based on a Fourier combination of velocity fields. Airy assumes the wave to form a sinusoidal elevation, but larger waves tend to form a more peaked crest. Therefore the Fenton-wave is more accurate for these larger deterministic, sometimes called higher order, waves. However the Airy-wave is able to be superpositioned to form stochastic wave spectra, which is not possible for the Fenton wave.
Based on this assessment, the Fenton wave is used for all deterministic waves defined by the wave period $\tau$ and the wave height $H$ within this research. For stochastic waves, the Airy wave is superimposed based on the JONSWAP-spectra, [8], defined by a peak Period $T_{P}$ and the significant wave height $H_{S}$.

To distinguish the overall resulting velocity field between the wake and velocity undulation from the periodic field of the waves, a baseline velocity $\vec{u}_{\text {baseline }}$ is defined according to Eq. (16). This velocity holds the constant Eulerian velocity $v_{1}$ for an undisturbed wave field resp. for an ACD the wave compensated local velocity in each time step.

$$
\begin{aligned}
& \vec{u}_{\text {baseline }}(t)=\vec{u}(t)-\vec{u}_{\text {wave }}(t)+v_{1} \\
& a_{\text {def-baseline }}=1-\frac{u_{\text {baseline }}}{v_{1}}
\end{aligned}
$$

\subsection{VIRTUAL FREE SURFACE}

For increased performance of the simulations, the free surface is not simulated in detail within this research. Instead the velocity vectors are calculated based on the wave theory at the mean sea level and applied as boundary condition to this location. Due to these boundary velocities the velocity field within the domain is equivalent to the field results of the wave theory as shown in Fig. 5. This method is called Virtual Free Surface (VFS) and described in detail in [9].

Fig. 6 enumerates the relative error of the VFS given by the baseline deficit $a_{d e f-\text { baseline }}$. As can be seen, the error is well below $1 \%$. The VFS has been limited to the region ahead and up to 10D behind the ACD to prevent numerical problems at the outlet of the domain. In this sector the VFS decays to the outlet at $x / D=30$, as can be seen in Fig. 6 at $x / D=15$.

An interesting anomaly compared to the classic free surface modeling in CFD is, that a 2 phase flow is simulated with a single phase. Therefore the wave through is filled with water instead of air. This seems to be a problem for the momentum equilibrium at the first glance, but due to the boundary condition pumping in and sucking out the water from the domain, the flow field fulfills the wave theory.

The method has been verified, [9], by direct comparison of 2 phase free surface and VFS simulations. It has been shown, that it is valid for fully submerged objects within a very small margin, but requires a modification for pressure field dominated loads. This is not the case here for the ACD.

However the VFS accurately predicts the wave to ACD interaction, it lacks the vice versa interaction of ACD to wave. With an increasing blockage of the current, the wave theory is not anymore representing the correct flow. Therefore the value of baseline deficit $a_{\text {def-baseline }}$ increases, indicating an error. Given the verification of [9], this is a lack in the definition of the baseline velocity, but not an error in the velocity field.

Therefore the drawback is assumed to be acceptable compared to the reduction of required resources for the simulation with a factor beyond 20. So the VFS is used for all wave simulations within this paper. 


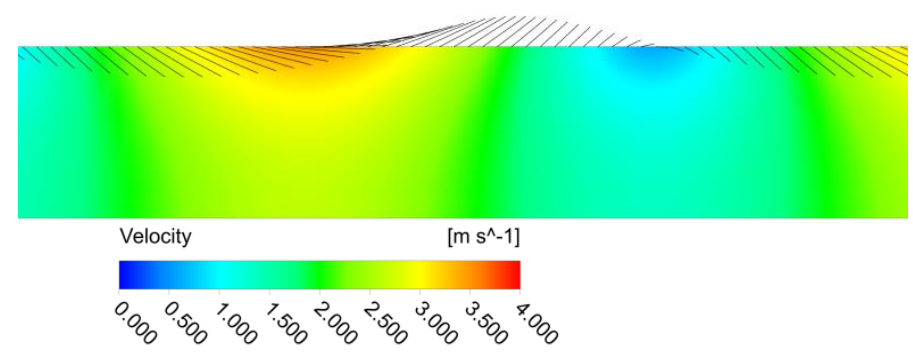

Fig. 5: Surface velocity vectors on VFS

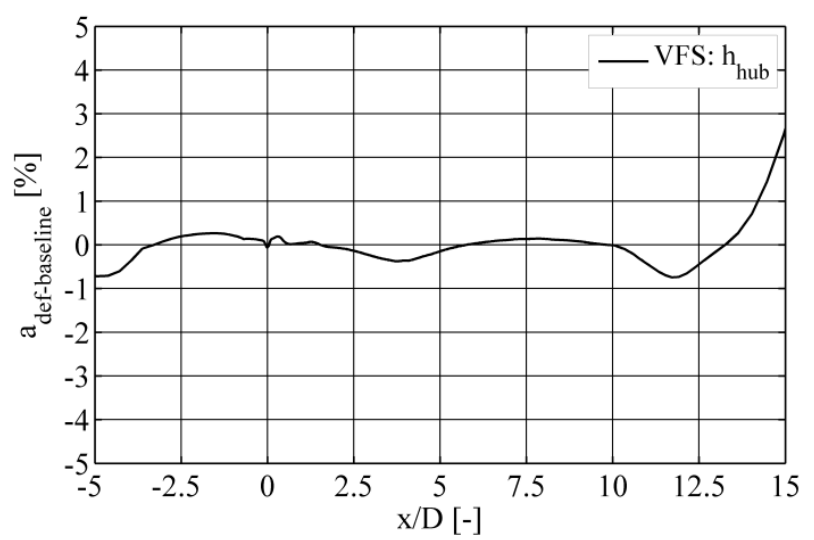

Fig. 6: Relative error in baseline velocity in undisturbed VFS

\subsection{MESH SETUP}

All simulations within this paper are performed on structured 2d-quad grids. The grid layout was based on an $\mathrm{H}-$ grid-topology as shown in Fig. 7. Compared to a 2 phase simulation, the grid resolution at the sea surface can be rather coarse, reducing the number of required elements, without losing accuracy or facing numerical damping of the waves. In the area ahead of the ACD and beyond a distance of $10 D$, the grid is also coarsened.

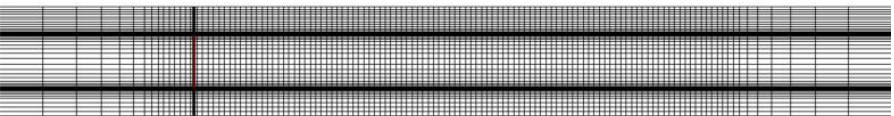

Fig. 7: Mesh coarsened by $1 / 3$

\subsubsection{ACD MESH}

Special attention has to be paid to the grid in vicinity of the ACD. The ACD needs to be thin compared to the extension $D_{A C D}$, according to the underlying assumptions. However reducing the thickness requires an increase of the source terms as outlined above and subsequent might result in numerical instabilities. Therefore a thickness $\Delta x_{A C D}=0.1 \mathrm{~m}$ was used.

Another problem of the grid in the vicinity of the ACD is that source terms in CFX can only be applied to the quadelements in the preprocessing of the simulation. The CFXSolver on the contrary solves the Navier-Stokes-Equations on virtual elements formed around each vertice. Therefore the source term is applied to half an element causing the solver to predict an unphysical velocity jerk. To prevent this, the beginning and end of the ACD volume needs to consist of very thin elements with $1 \mathrm{~mm}$ thickness here. To limit the total number of elements the volume change in this sector is chosen to be large with values between 1.5 and 2 .

Further the tip area of the ACD has been refined to accurately predict the turbulent shear layer between free stream and wake. The resulting grid topology of this sector is shown in Fig. 8.

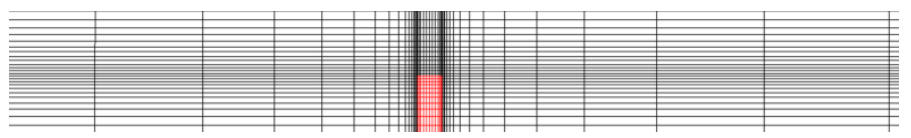

Fig. 8: Mesh in vicinity of ACD

\subsubsection{NUMERICAL WAKE DAMPING}

To check for numerical damping due to the mesh, a refinement study for the wake sector has been carried out. For a wave with $H=4 m$ and $\tau=8 \mathrm{~s}$ in a current with $v_{1}=2 \frac{\mathrm{m}}{\mathrm{s}}$ and water depth $D=30 \mathrm{~m}$ the wake deficit of a ACD with $c_{x}=2$, $D_{A C D}=15 \mathrm{~m}$ and $h_{h u b}=15 \mathrm{~m}$ is shown in Fig. 9 for 3 axial grid resolutions. As can be seen, the wake is near to identical for all 3 cases.

Although the simulation time increases with number of grid elements, the medium grid resolution is used. With a total of $80 \mathrm{k}$ elements and a simulation time of approx. $2 \mathrm{~h}$ on 8 Cores for 25 wave periods, it requires reasonable low resources. Further reduction of the grid resolution would give a larger uncertainty regarding the grid independency for varying flow parameters.

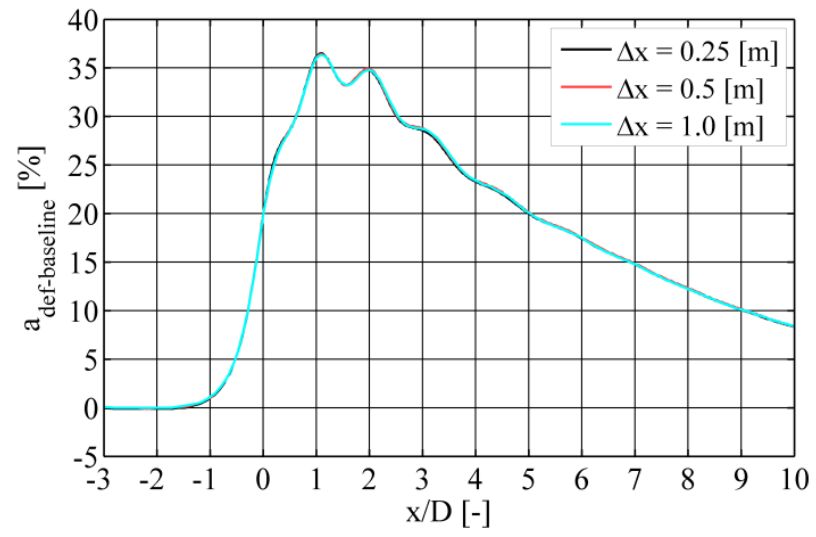

Fig. 9: Centerline baseline wake deficit with varying grid resolutions

\section{RESULTS}

To analyze the velocity undulations and its effects, a wide range of model parameters are varied. The results of these simulations are shown and detailed below.

The calculated centerline wake results are shown by means of the ratio given in Eq. 18. This value is evaluated over the final 2 wave periods of each simulation and represents the increase of velocity amplitude with undulations compared to 
the apparent wave field. It correlates therefore with the relative increase of loads.

$$
\begin{aligned}
& \frac{\Delta u_{\text {baseline }}}{\Delta u_{\text {wave }}}=\frac{\max \left(u_{\text {baseline }}\right)-\min \left(u_{\text {baseline }}\right)}{\max \left(u_{\text {wave }}\right)-\min \left(u_{\text {wave }}\right)} \\
& \frac{\Delta u_{\text {baseline }}}{\Delta u_{\text {wave }}}=\frac{\max \left(u-u_{\text {wave }}\right)-\min \left(u-u_{\text {wave }}\right)}{\max \left(u_{\text {wave }}\right)-\min \left(u_{\text {wave }}\right)}
\end{aligned}
$$

\subsection{FORMATION OF VELOCITY UNDULATIONS}

The formation of the velocity undulations is dependent on the timing of the wave. As shown in Fig. 10 the undulation develops over time within a distance of 1D behind the turbine. On $t / \tau=0.166$ at top most position of the ACD a sector with low velocity occurs and moves to the centerline of the turbine, $t / \tau=0.333$. At this location it merges with the centerline wake, $t / \tau=0.666$, and forms a symmetric velocity undulation moving on with the wake. This process then repeats with the next wave.

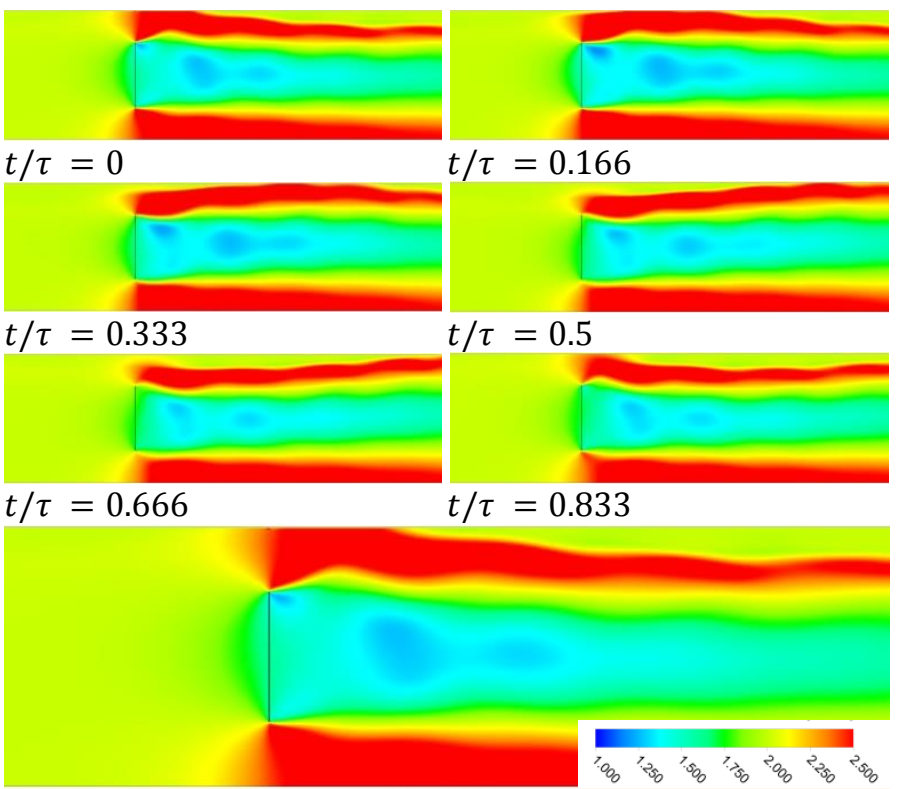

$t / \tau=1$

Fig. 10: Formation of the velocity undulation in baseline velocity $u_{\text {baseline }}\left[\frac{\mathrm{m}}{\mathrm{s}}\right]$ (case: $H=4 \mathrm{~m}, \tau=8 \mathrm{~s}, \quad v_{1}=2 \frac{\mathrm{m}}{\mathrm{s}}$, $D=30 m, c_{x}=2$ )

This location at the top most position of the ACD as source of the velocity undulation could be expected due to the penetration depth of the wave and the high velocity amplitude $\Delta u_{\text {wave }}$. However the TKE differs from this behavior in the velocity field as can be seen in Fig. 11. The TKE is of the same order of magnitude at the top and bottom end of the ACD. However also in the TKE areas of increased and reduced intensity occur.

This is a result of the vertical component of the wave orbital velocities as shown in Fig. 12 by particles starting at the tips of the ACD and moving with the flow. Over time the particles are converging to groups, equivalent to the areas of increased TKE.
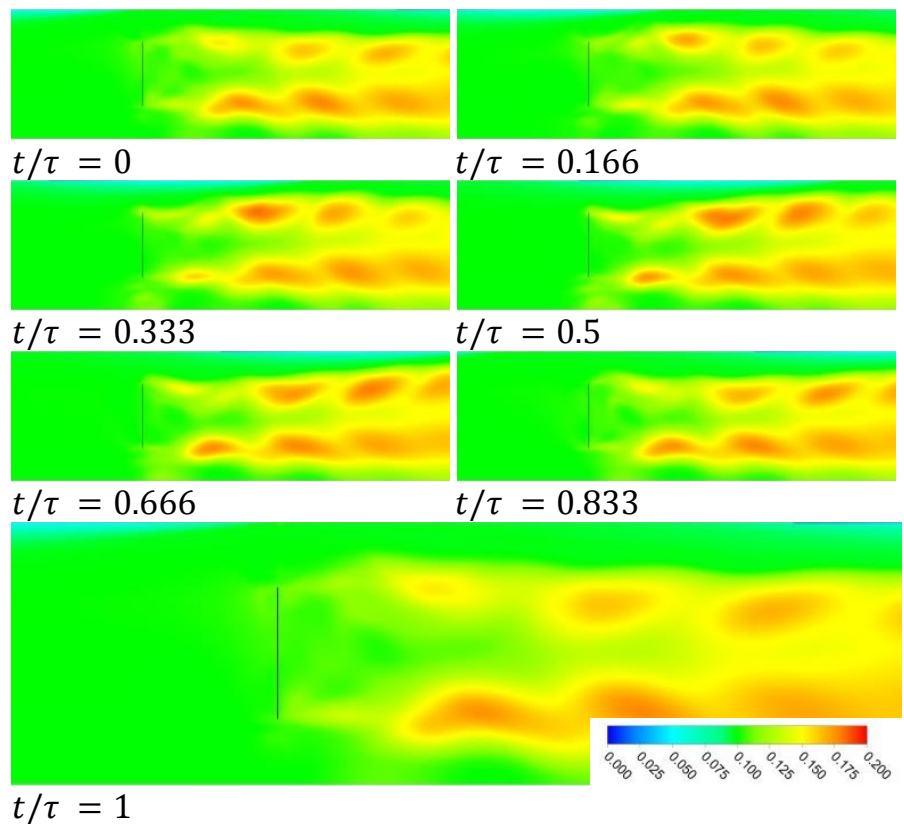

Fig. 11: Formation of the velocity undulation in turbulence intensity $I$ (case: $H=4 m, \tau=8 s, v_{1}=2 \frac{m}{s}, D=30 m, c_{x}=$ 2)

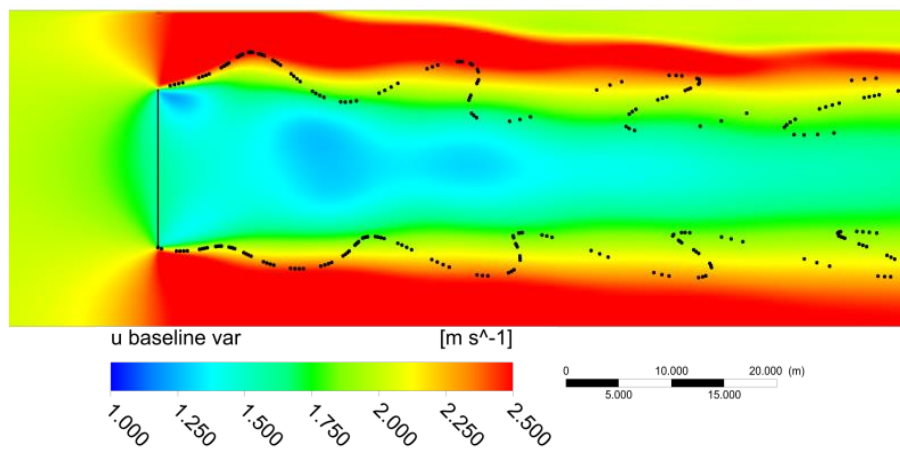

Fig. 12: Momentaneous position of particles emitted at blade tips over time (case: $H=4 m, \tau=8 s, v_{1}=2 \frac{m}{s}, D=30 m$, $\left.c_{x}=2\right)$

\subsection{IMPACT OF CURRENT PARAMETERS}

Within this section the effect of current parameters are investigated. These cover the Eulerian current velocity $v_{1}$ and the water depth $D$.

\subsubsection{CURRENT}

The results of the variation of current velocity is shown in Fig. 13 for the values $v_{1}=2 \frac{\mathrm{m}}{\mathrm{s}}, v_{1}=4 \frac{\mathrm{m}}{\mathrm{s}}$ and $v_{1}=6 \frac{\mathrm{m}}{\mathrm{s}}$. This is the typical range of values occurring at tidal energy sites. As can be seen there are 3 differences occurring on the cases. On one hand, the positions of the destructive interference occur for 
higher flow speeds on much more distant locations. This effect is nonlinear over $v_{1}$ and $x / D$. It can be explained by the higher velocities in the wake moving the undulations and a constant speed of waves. The nonlinearity occurs due to the motion with the local velocity and the finite length of the wake of the ACD.

On the other hand the actual magnitude and the damping of the velocity undulations also change with $v_{1}$. As can be seen, the damping decreases, which is a result of the higher energy and therefore higher stability in the flow. However the increase of the magnitude can not be explained by the increased energy in the flow, but by the increased induced velocity, which result in stronger sector of reduced speed, cf. 3.1.

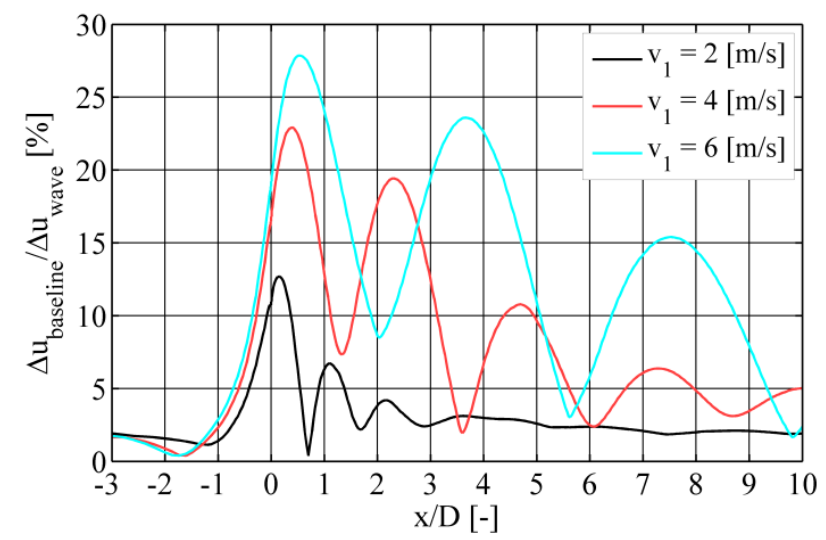

Fig. 13: Relative amplitude increase of axial velocity in turbine wake on current variation (case: $H=4 m, \tau=8 s, D=45 m$, $c_{x}=2$ )

\subsubsection{WATER DEPTH}

For the water depth $D$ a smaller variation in results occurs as shown in Fig. 14. For all cases the order of magnitude of the velocity undulations is the same. However due to the relative definition in the diagram, the absolute value of velocity undulation is much smaller for increased $D$.

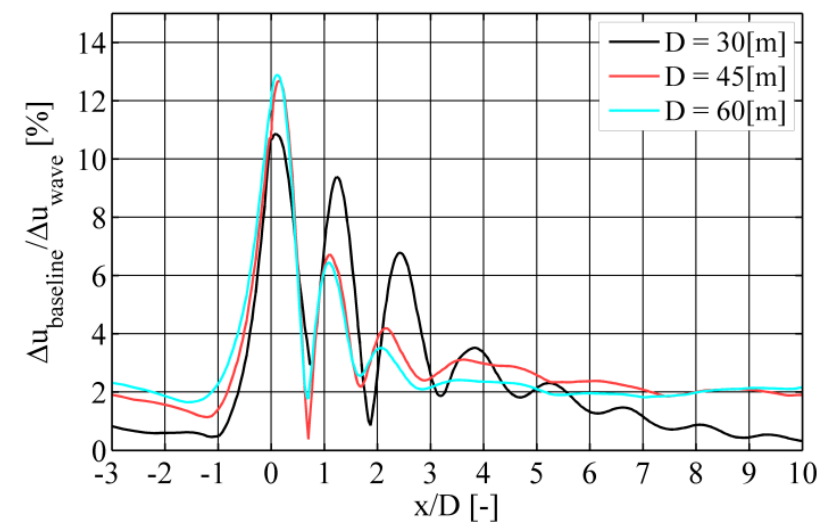

Fig. 14: Relative amplitude increase of axial velocity in turbine wake on water depth variation (case: $H=4 m, \tau=8 s$, $\left.v_{1}=2 \frac{m}{s}, c_{x}=2\right)$
Despite the approx. same magnitude of the velocity undulations, the damping is increased for higher water depths. This is a result of the reduced gradient $\frac{d u_{\text {wave }}}{d z}$ for higher $D$, leading to the apparent higher damping. Therefore the results of $D=45 \mathrm{~m}$ and $D=60 \mathrm{~m}$ are near to the same, as the gradient $\frac{d u_{\text {wave }}}{d z}$ is approx. constant for high water depths.

\subsection{IMPACT OF WAVE PARAMETERS}

Waves are defined by their height, period and direction. While height and period change the shape of the wave and subsequent the velocity undulations, the direction impacts the alignment of wake and axial direction.

The impact of this alignment to the velocity undulations is uncertain, but expected to be small compared to the wave shape. However to investigate these changes, a 3-dimensional simulation with significantly increased computational resources is required. Therefore only the wave shape is varied here.

\subsubsection{WAVE HEIGHT}

The wave height is a parameter to amplify any wave dependent loads. However as shown in Fig. 15 it is due to the relative definition of minor relevance here. Except the increased uncertainty in the velocity field due to the definition of $u_{\text {baseline }}$ and the VFS rising to up to $3 \%$ for $H=1 \mathrm{~m}$, the velocity undulation is the same for all investigated cases.

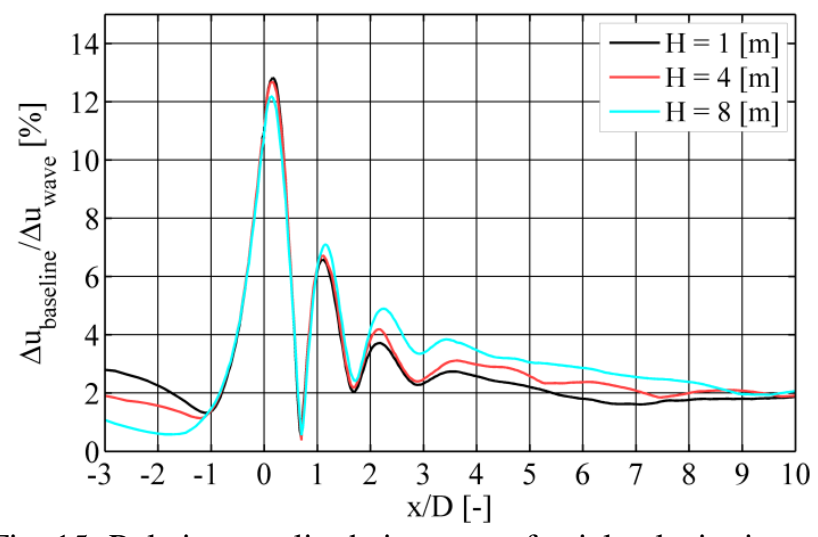

Fig. 15: Relative amplitude increase of axial velocity in turbine wake on wave height variation (case: $\tau=8 \mathrm{~s}, v_{1}=2 \frac{\mathrm{m}}{\mathrm{s}}$, $D=45 m, c_{x}=2$ )

\subsubsection{WAVE PERIOD}

With rising wave period the velocity undulations grow up as shown in Fig. 16. Also a change in wave velocity can be noticed and therefore a change of the locations of interference. However only for $\tau=8 \mathrm{~s}$ a significant destructive interference is visible. The reason for this difference could not be found within this research. 


\subsection{IMPACT OF TURBINE CHARACTERISTIC}

The characteristics of a tidal current turbine are governed by a large number of parameters; however most of them are overridden by the assumption of the ACD type. Therefore only the thrust coefficient $c_{t}$, channel blockage and turbine control are investigated here.

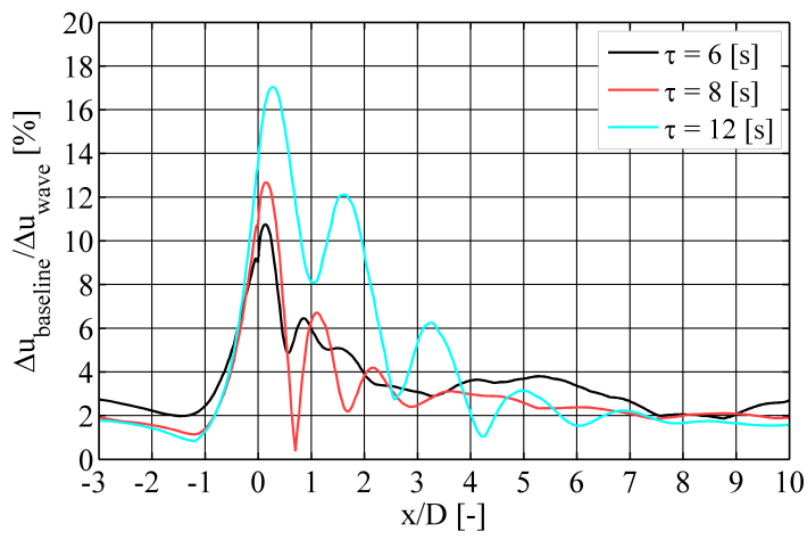

Fig. 16: Relative amplitude increase of axial velocity in turbine wake on wave period variation (case: $H=4 m, v_{1}=2 \frac{\mathrm{m}}{\mathrm{s}}$, $D=45 m, c_{x}=2$ )

\subsubsection{THRUST COEFFICIENT}

The thrust coefficient is defined by the point of operation of the turbine. For low flow velocities it is to be expected near to the Betz optimum $c_{t}=8 / 9$, as the turbine operates for maximum power production. With increasing flow velocity the thrust coefficient might be lowered for above rated operation resulting in a reduction of the velocity undulations as outlined by Fig. 17 .

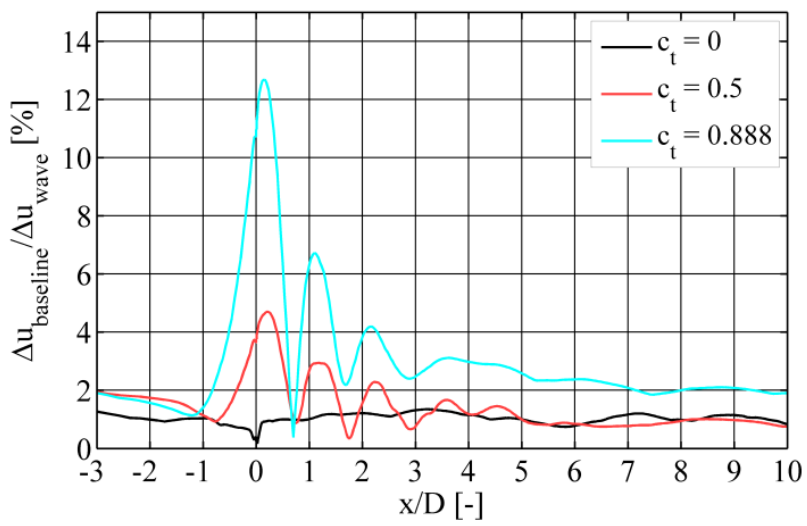

Fig. 17: Relative amplitude increase of axial velocity in turbine wake on thrust coefficient variation (case: $H=4 m, \tau=8 s$, $v_{1}=2 \frac{m}{s}, D=45 m$ )

\subsubsection{BLOCKAGE}

To maximize the power output of a given site, the blockage of the flow can be increased. This is done here by varying the vertical size of the ACD $D_{A C D}$ within the 2-dimensional channel of constant depth. In Fig. 18 the resulting velocity undulations are shown. In difference to the other results it is shown here over the absolute distance of the wake to compare the damping and interference behavior.

As can be seen, the absolute locations of the destructive interference remain the same. It is also interesting, that despite a massive increase of the $1^{\text {st }}$ velocity undulation, the $2^{\text {nd }}$ and $3^{\text {rd }}$ undulations are of the same order of magnitude for all cases.

However for a reduced size of tidal current turbines, the turbines need to be placed with a lower absolute distance to maintain the power of the park. Therefore the increased first velocity undulation will reach the $2^{\text {nd }}$ row with a higher impact compared to a large turbine.

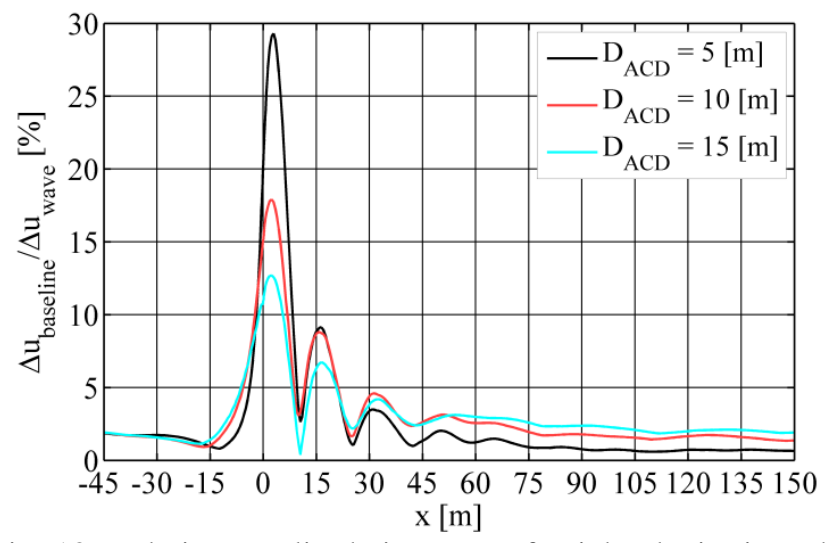

Fig. 18: Relative amplitude increase of axial velocity in turbine wake on ACD diameter $D_{A C D}$ variation over absolute distance (case: $H=4 m, \tau=8 s, v_{1}=2 \frac{m}{s}, D=45 m$ )

\subsubsection{TURBINE CONTROL}

The turbine control is the main parameter for the designer to adapt the turbine to improve behavior. As shown in Fig. 19 the type of the ACD has a significant impact to the velocity undulations in the wake. A reduction by $60 \%$ of the added velocity amplitude could be achieved.

The $c_{x^{-}}$and $F_{x}$-ACD are symbolic here for 2 controller strategies. While the $c_{x}$-ACD is a turbine with zero response time and maintains always the optimal rotational speed resp. tip speed ratio $\lambda_{\text {opt }}$, the $F_{x}$-ACD is a load controlled turbine aimed to hold the absolute rotor thrust constant. However the $c_{x}$-ACD is the theoretic optimum for power production and can be achieved for a light enough turbine, the $F_{x}$-ACD is a pure theoretic turbine and its behavior is hard to achieve for a real turbine.

An intermediate model is therefore an $c_{x}$-ACD with a finite response time. This is achieved within this research by applying a $P T_{1}$-low pass filter to the input value $u$ of the ACD to form the $c_{x 1}$-ACD. This can be described in time discrete formulation as done in Eq. (20) - (22).

In Fig. 20 the results for a variation of the time parameter $T_{1}$ of the $P T_{1}$-element is shown. As can be seen, a reduction of 
the swiftness of the control sequence of a tidal current turbine can reduce the velocity undulations and therefore improve the park behavior of the turbines.

$$
\begin{aligned}
& T^{\star}=\frac{1}{\frac{T_{1}}{d t}+1} \\
& \mathrm{u}_{\mathrm{PT}_{1}}^{2}=T^{\star}(u(t)-u(t-d t))+u(t-d t) \\
& \Delta p_{P T 1}=\frac{\rho}{2} \cdot u_{P T_{1}}^{2} \cdot c_{x}
\end{aligned}
$$

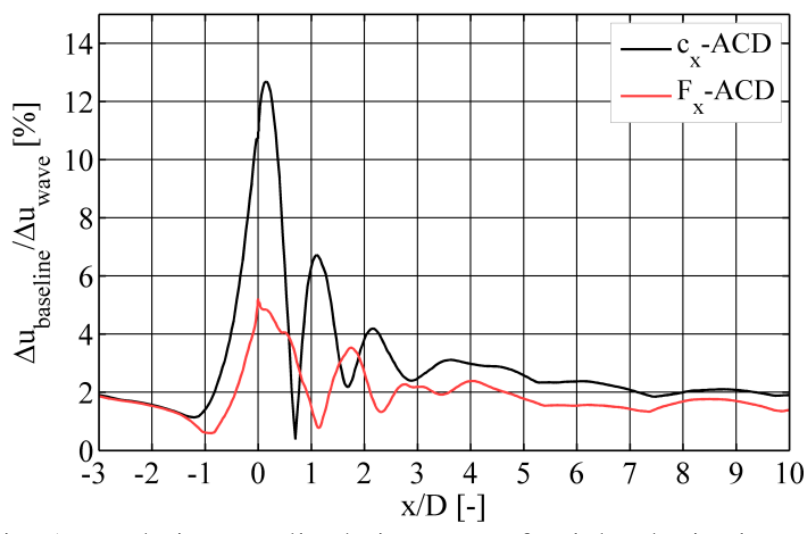

Fig. 19: Relative amplitude increase of axial velocity in turbine wake on controller target variation (case: $H=4 m, \tau=8 s$, $v_{1}=2 \frac{m}{s}, D=45 m, c_{x}=2$ )

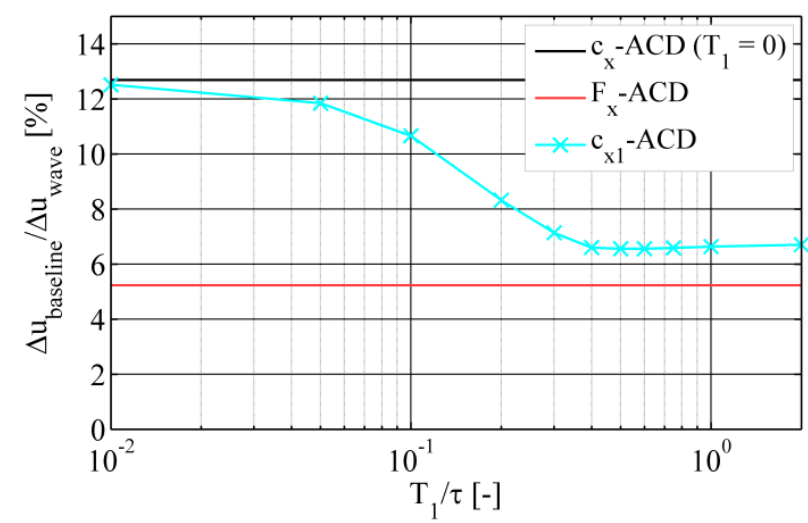

Fig. 20: Peak relative amplitude increase of axial velocity in turbine wake on controller swiftness variation (case: $H=4 m$, $\tau=8 s, v_{1}=2 \frac{m}{s}, D=45 m, c_{x}=2$ )

\subsection{STOCHASTIC WAVES}

Ocean waves are seldom deterministic as assumed in the investigation above. Therefore the more realistic case of a stochastic wave has been simulated in addition here. The waves are therefore a superposition of linear waves to form a JONSWAP-spectra with a peak enhancement factor $\gamma=3.3$ and $\gamma=7$. These values cover the normal range of ocean waves.
To cover the effect of the random phases, a total of $800 \mathrm{~s}$ has been simulated, with the later 600 s for analysis and post processing.

As shown in Fig. 21 by comparison to the equivalent deterministic wave, the velocity undulation and subsequent the velocity amplitude in wake still occurs. However the uncertainty of the definition of $u_{\text {baseline }}$ as outlined in 2.5 increases here to $5 \%$. Nevertheless the magnitudes and damping of the cases are roughly the same.

An interesting difference is the reduction of destructive interference. This is a result of the combination of different wave periods and wave speeds. However, the positions of these interference locations are dependent on multiple parameters as shown above and of minor importance.

Therefore the results of the deterministic waves given above can be translated to the more general stochastic waves shown here.

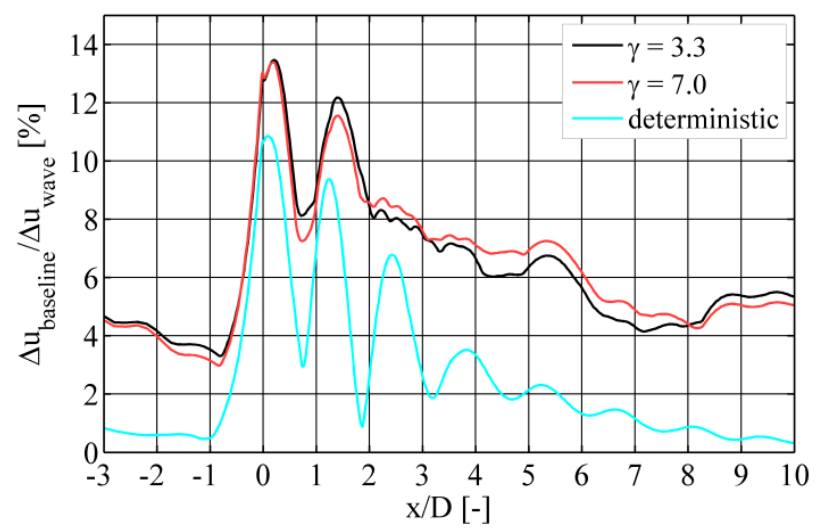

Fig. 21: Relative amplitude increase of axial velocity in turbine wake on stochastic waves with peak enhancement factor $\gamma$ variation (case: $H=H_{S}=4 m, \tau=T_{P}=8 s, \quad v_{1}=2 \frac{m}{s}$, $\left.D=30 m, c_{x}=2\right)$

\section{CONSEQUENCES FOR PARK LAYOUT}

Based on the above findings multiple consequences for the optimal park layout can be drawn. On one hand, the wave loads significantly increase for a to small turbine spacing. Therefore the spacing needs to be large enough, not only to increase the absolute power output of the park, but also to limit the wave loads. From the above findings no distinct spacing can be drawn, but a lower value than 5D increases the risk of increased loads. This value is obtained by comparison of above results, showing, that for each case the undulations are damped out within approximately 5D except for extreme flow velocities.

On the other hand, larger turbine diameters fit the requirements of parks regarding loads better. As shown above for the impact of the blockage, 3.4.2, smaller turbines result in stronger undulations with the same absolute distance of damping. Therefore the turbines either need to be built heavy enough to withstand the additional loads from the velocity undulations, or the spacing needs to be increased beyond economic limits. 
Within the design process of tidal current turbines for park application an additional safety factor on the wave conditions of $j_{\text {undulation }}=1.3$ is recommended based on the highest observed velocity undulations in the investigation above. This value might be reduced if the park is analyzed for the actual size and occurrence of the velocity undulations. During this analysis the turbine size, response time, current and wave condition needs to be modelled according to the range of conditions at site.

However during the design process of the turbines the turbine controller can be used to prepare the turbine for park application. For high current speeds the controller needs to be slower than half the significant wave period, typically $T_{1}>4 \mathrm{~s}$, to reduce the undulations on the $2^{\text {nd }}$ row of turbines as shown in 3.4.3. For reduced current speeds, the undulations move over a shorter distance and the response time might be decreased resulting in lower $1^{\text {st }}$ row turbine loads. This change in individual control target might be accomplished by a supervisory park controller.

\section{CONCLUSIONS}

The present paper deals with velocity undulations in the wake of tidal current turbines under wave influence. It has been shown, that these velocity undulations are a result of the difference between wave speed and current velocity leading to a displacement in the energy field.

The effect of velocity undulations has been investigated in detail and the influence of a wide range of operational parameters has been shown. An increase of approx. $10 \%$ in wave loads is to be expected for default park parameters, but might increase well beyond $30 \%$ added loads for ill-suited park setups.

The paper concluded with a first view on the consequences for park setups with respect to surface waves and the importance of the control strategy for park applications.

\section{ACKNOWLEDGMENTS}

This project at the University Stuttgart and Stuttgart Chair of Wind Energy (SWE) is funded by Voith Hydro Ocean Current Technologies $\mathrm{GmbH} \& \mathrm{Co}$. KG. Further it is supported by the software suppliers Simpack AG and Ansys Germany GmbH.

\section{REFERENCES}

[1] Ansys, 2014, "Ansys CFX - Theory Guide", Software documentation

[2] Arnold, M., 2011, “Untersuchung einer Gezeitenströmungsturbine und Haltestruktur mittels strömungsmechanischer Simulation", Thesis, University Stuttgart

[3] Batten, W., Harrison, M., Bahaj, A., 2013, "The accuracy of the actuator disc-RANS approach for predicting the performance and far wake of a horizontal axis tidal stream turbine", Philosophical Transactions of The Royal Society A Mathematical Physical and Engineering Sciences, Vol. 371, pp. 1-15.
[4] Crespo, A., Hernández, J., 1996, “Turbulence characteristics in wind-turbine wakes", Journal of Wind Engineering and Industrial Aerodynamics, Vol. 61, pp. 7185 .

[5] Faudot, C., 2012, "Prediction of Wave Loads on Tidal Turbine Blades”, Energy Procedia, Vol. 20, pp. 116-133

[6] Fenton, J., 1988, "The numerical solution of steady water wave problems", Computers \& Geosciences, Vol. 14, pp. 357-368

[7] Garder, D., 2013, "Untersuchung des dynamischen Verhaltens des Nachlaufs von Gezeitenströmungsturbinen unter Welleneinfluss", Thesis, University Stuttgart.

[8] Hasselmann, K., et al., 1973, "Measurements of windwave growth and swell decay during the Joint North Sea Wave Project JONSWAP”, Ergänzungsheft zur Deutschen Hydrographischen Zeitschriften Reihe, Vol.8, p. 95

[9] Markus, D., Arnold, M., Wüchner, R., Bletzinger, K.-U., 2014, “A Virtual Free Surface (VFS) model for efficient wave-current CFD simulations of fully submerged structures", accepted by Journal of Coastal Engineering.

[10] Menter, F., 1993, “Zonal Two Equation k- $\omega$ Turbulence Models for Aerodynamic Flows", AIAA, Paper 93-2906.

[11] Mycek, P., Gaurier, B., Germain, G., Pinon, G., Rivoalen, E., 2013, "Numerical and Experimental Study of the Interaction Between Two Marine Current Turbines", International Journal of Marine Energy, Vol. 1, pp. 70-83.

[12] Myers, L., Bahaj, A., 2010, "Experimental analysis of the flow field around horizontal axis tidal turbines by use of scale mesh disk rotor simulators", Journal of Ocean Engineering, Vol. 37, pp. 218-227.

[13] Simon, T., 2012, "Simulation poröser Medien in einem Wasserkanal mit freier Oberfläche", Thesis, University Stuttgart

[14] Stallard, T., Collings, R., Feng, T., Whelan, J., 2011, "Interactions Between Tidal Turbine Wakes: Experimental Study of a Group of 3-Bladed Rotors", Proceedings of $9^{\text {th }}$ European Wave and Tidal Energy Conference, Southampton, UK.

[15] Spalart, P., Rumsey, C., "Effective Inflow Conditions for Turbulence Models in Aerodynamic Calculations", 2007, AIAA Journal, Vol. 45, pp. 2544-2553

[16] Whelan, J., Thomson, J., Graham, J., Peiró, J., 2007, "Modelling of free surface proximity and wave induced velocities around a horizontal axis tidal stream turbine", Proceedings of $7^{\text {th }}$ European Wave and Tidal Energy Conference, Porto, Portugal 
